\title{
Hepatic Perfusion Disorders Secondary to Superior Vena Cava Obstruction: Two Cases Reports
}

\author{
A. Benhima*, M Ait Chtouk F, Amenzouy M, Ouali Idrissi N, Cherif Idrissi Gannouni
}

Radiology Department, ARRAZI Hospital, Mohammed VI th University Hospital Center, Cadi Ayad University, Marrakesh, Morocco

DOI: $10.36347 /$ simcr.2020.v08i08.015

| Received: 09.08.2020 | Accepted: 17.08.2020 | Published: 28.08.2020

*Corresponding author: Benhima Anouar

Abstract

Hepatic perfusion disorders are focal or diffuse abnormalities of the parenchymal enhancement after injection of contrast agent. Superior vena cava thrombosis disease is relatively rare due in most cases to external compressions of the superior vena cava (SVC) by a mediastinal neoplasm: lymphadenopathy, mediastinal tumor. Several venous collateral pathways usually can develop allowing blood to return to the right atrium such us systemic-portal-shunting which are rarely involved. This leads to an increase flow sat the lower vein cava and the liver. We will expose in this work through two cases of thrombosis of the superior vena cava, the development mechanisms of the various collateralities involved creating systemo-portal anastomoses at the origin of these perfusion disorders as well as their translation in computed tomography.

Keywords: Hepatic perfusion disorders, superior vena cava obstruction, side venous circulation, triangular contrast enhancement, systemo-portal bypass, segment IV.

Copyright @ 2020: This is an open-access article distributed under the terms of the Creative Commons Attribution license which permits unrestricted use, distribution, and reproduction in any medium for non-commercial use (NonCommercial, or CC-BY-NC) provided the original author and source are credited.

\section{INTRODUCTION}

Upper vena cava thrombosis is a rare pathology secondary most often to compression or invasion by a mediastinal tumor process or more rarely to thrombosis on a central catheterization: the venous return to the right cardiac chambers is ensured by venous collateralities [4,5] including systemo-portal bypass pathways. This is secondary to an increase in flows in the inferior vena cava and the liver as demonstrated by the results of the cava venography and scintigraphy $[1,6,7]$. helical computed tomography (CT) highlights early focal contrast enhancements in the hepatic areas where vascular flow is increased. We illustrate in this work the cases of two patients with a thrombosis of the SVC and in whom the CT scan showed abnormalities of the hepatic perfusion. We insist on the form and the localization of these focal contrasts and on the venous collateralities involved.

\section{CASe 1}

A 50-year-old woman followed for breast cancer with bone metastasis. A thoraco abdominopelvic CT scan was performed as part of its extension assessment. The liver was explored by three phases (hepatic arterial phase, portal venous phase and tardive phase) acquired respectively at 25, 70 and 180 seconds after contrast injection. It revealed a stenotic of the right and left brachiocephalic vein trunks with partially obstructive thrombosis of the SVC and development of a paravertebral collateral vein, the lateral thoracic vein, associated to significant expansion of the azygos vein and internal mammary veins. It also showed, after contrast injection at arterial phase, an intense, triangular, sub capsular contrast, limited to the left hepatic lobe and a part of segment IV of the liver related with a side venous circulation between the left portal branch and the epigastric vein, they are supplied by collateral from the internal mammary with a venous return joining the median hepatic vein (fig 1 and 2 ). Liver ultrasound was normal.

\section{CASE 2}

A 59-year-old man without previous medical history consulted for right lateral cervical mass and face oedema. The clinical examination revealed a superior vena cava syndrome.

The thoracoabdominal CT showed an enlarged mediastinal lymphadenopathy compressing the ascending aorta, the arch and the supra-aortic trunks, the SVC and the innominate trunks with extensive thrombosis of both internal jugular veins. 
During arterial injection, the CT scan showed a fleeting and triangular contrast enhancement of the superior part of segment IV that disappeared during the arterial phase (fig 3 and 4). Liver ultrasound was normal.

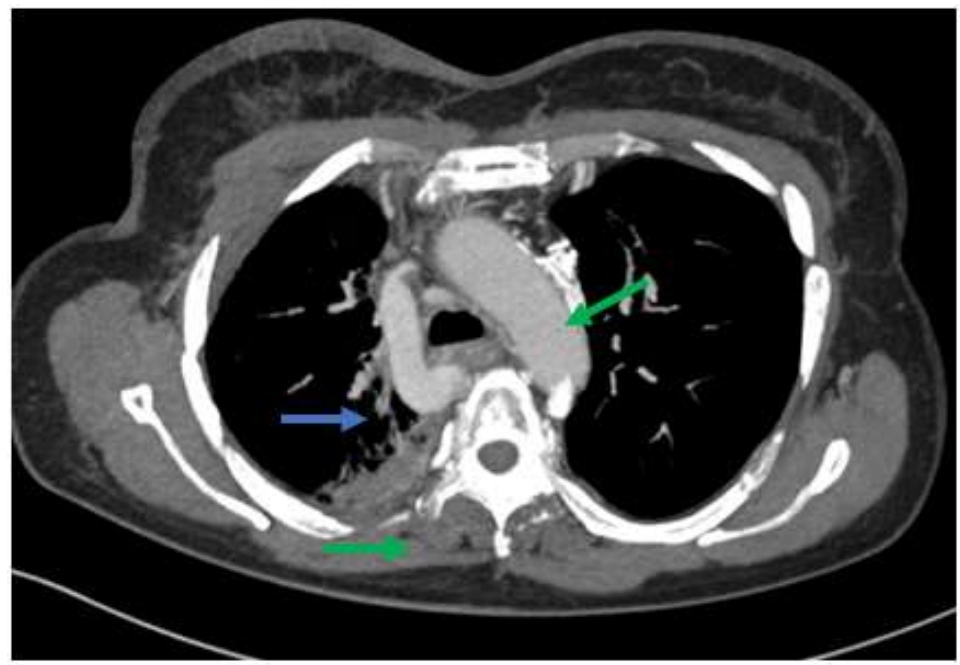

Fig-1: Contrast-enhanced axial CT of the chest: Mediastinal window Dense enhancement and dilation of the azygos arch (blue arrow) with mediastinal and peri-spinal veins collaterals (green arrow)

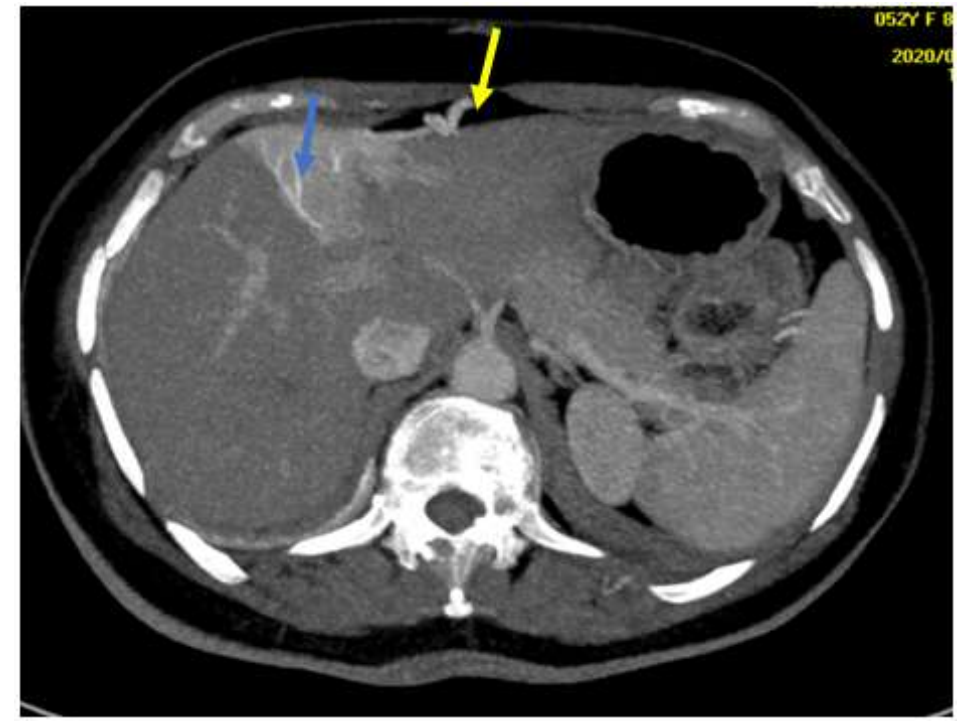

Fig-2: Contrast-enhanced CT scan of the liver during the arterial phase Hyperdense enhanced area within segment IV of the liver (green arrow). Dilation of the epigastric vein (yellow arrow)

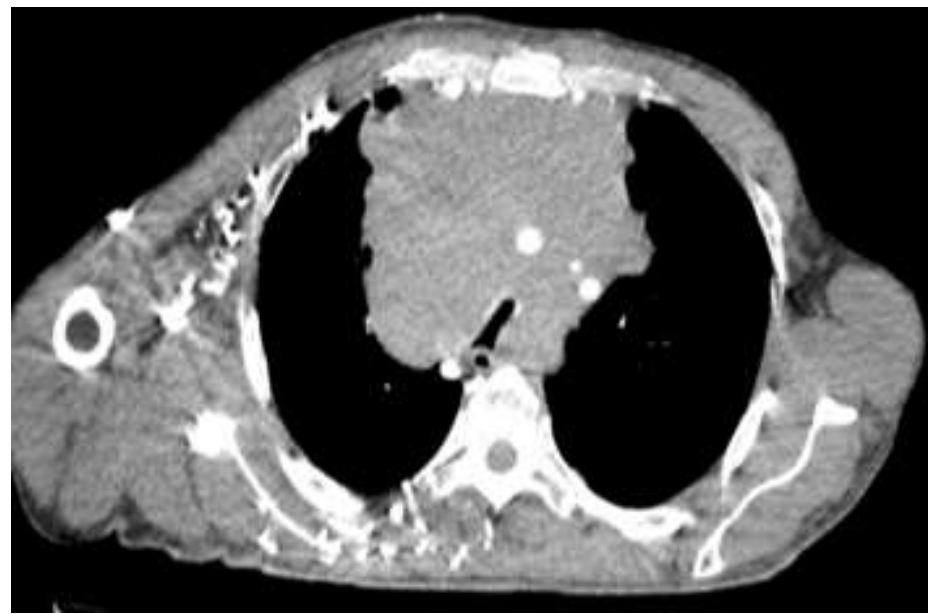

Fig-3: Contrast-enhanced axial CT of the chest: Mediastinal window Enlarged compressive mediastinal lymphadenopathy responsible of vascular infiltration and thrombosis of the SVC with dilation of the azygos vein 


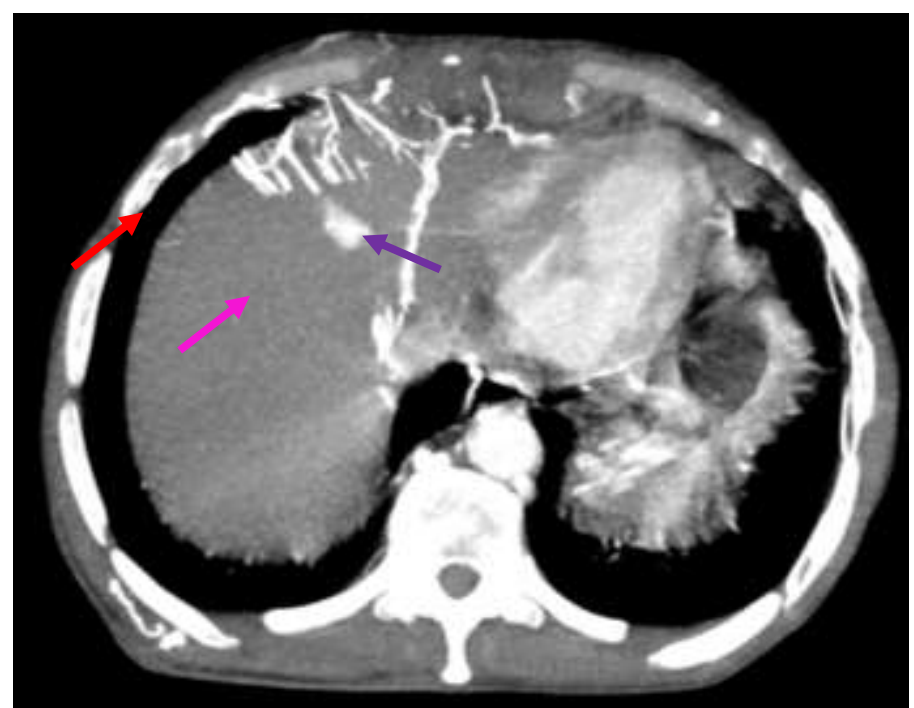

Fig-4: contrast-enhanced CT scan of the liver during the arterial phase: Hyperdense contrast enhancement in the superior part of segment IV (pink arrow) drained by dilated subcapsular veins (red arrow) to the phrenic vein (purple arrow)

\section{DISCUSSION}

Liver perfusion disorders are transient abnormalities in the enhancement of the healthy liver parenchyma. The main causes are venous obstructions, which can occur at any level: portal, sinusoidal or hepatic venous. Most authors [1-3] agree on the rarity of disorders of the hepatic perfusion in case of TCS. Imaging is fundamental for the diagnosis.

Through our two cases, we objectified the taking of intense and early focal contrasts in patients with TCS. These contrast enhancements are explained by the presence of system-portal shunts which pours into the liver the contrast product accumulated upstream of the thrombosis site $[1,2]$.

On CT scan, the contrast uptakes encountered in the liver are early, appearing at the arterial time of the injection. They are in badly limited, intense and ephemeral ranges. These contrasts are intense and early because it is pure contrast having not achieved a cardiac passage; CT also allows recognizing venous thrombosis, to specify its cause, its extent and its degree.

When there is a hepatic obstacle, particularly during a TCS, four main venous bypass routes are used $[1,6,7]$ :

- The azygos-hemi azygos system,

- The peri-spinal venous plexus

- The internal mammary vein,

- The lateral thoracic and thoraco-abdominal veins

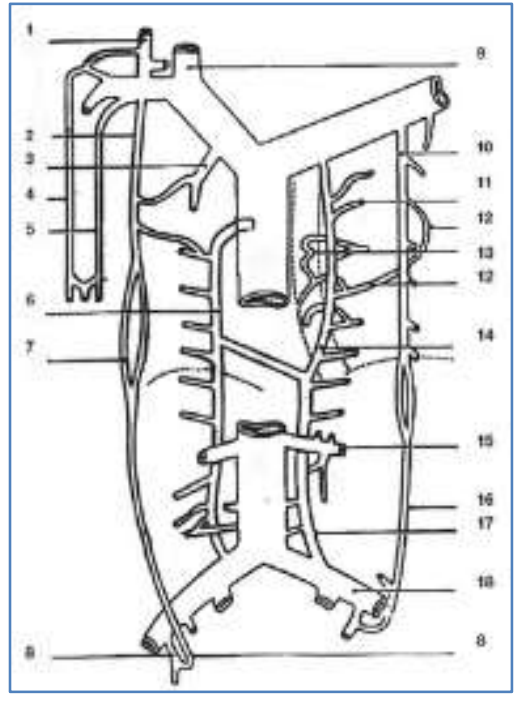

Fig-5: Vertical anastomoses. (a) Short and long vertical anastomoses. 1. External jugular vein. 2. Right external mammary vein. 3. Right internal mammary vein. 4 . Cephalic vein. 5. Basilic vein. 6. Venous azygos. 7. Superficial abdominal subcutaneous vein. 8. Long saphenous arch. 9. Right internal jugular vein. 10. Left internal mammary vein. 11. Posterior intercostal vein. 12. Anterior intercostal vein. 13. Esophageal vein. 14. Accessory hemi-azygos vein. 15. Left renal vein. 16. Left epigastric vein. 17. Ascending lumbar vein. 18. Left external iliac vein

- The azygos-hemiazygos system, formed by the azygos vein, the hemiazygos and accessory hemiazygos veins, constitutes a vast anastomotic network between the upper vena cava and the lower vena cava (fig. 5). It communicates with the internal mammary vein via the anastomoses connecting on the one hand the collateral anterior intercostal veins of the internal mammary vein and on the other hand the posterior intercostal veins which flow on each floor into the azygos system It also communicates with the peri-spinal venous plexus which, via the dorso-spinal veins, flows into the posterior intercostal veins $[7,8]$. 
- The perish-spinal venous network is like the azygos system in connection with the inferior vena cava. The upper and lower phrenic veins which flow into the internal mammary vein and which are in communication with the subcapsular veins of the liver could also be involved during TCS [6]. This path has been followed in our patients.

- The internal mammary vein communicates with the superior epigastric vein, the latter is in connection with the portal system via the peri-umbilical venous system which flows into the left portal branch. The internal mammary vein flows into the brachiocephalic venous trunk. With $\mathrm{CT}$ and in case of thrombosis of the VCS, the internal mammary vein becomes opaque against the current, directly by the brachiocephalic venous trunk upstream of the thrombosis and indirectly by its connections with the azygos system. The clouded blood then circulates in the superior epigastric vein which communicates with a recapitalized para-umbilical vein which drains into the left branch of the portal vein [9]. It should be noted that this type of cavoportal shunt can be functional also in the event of thrombosis of the inferior vena cava via the inferior epigastric vein which arises from the external iliac vein and anastomoses with the superior epigastric vein [9].

- The superficial lateral and thoraco-abdominal thoracic veins also communicate with the epigastric veins and the portal system. At CT, the lateral thoracic and thoraco-abdominal veins, superficial, homolateral at the site of the PDC injection, opacify against the current from the subclavian vein (fig. 5).

The contrast uptakes encountered in the liver are early appearing at the arterial time of the injection. They are in badly limited, intense and ephemeral ranges. These contrasts are intense and early because it is pure contrast which has not produced a cardiac passage. In the study by Baba et al. [6] the authors divide these hepatic contrast enhancements into four territories: the anterior part of segment IV, the subphrenic region, the posterior part of the right lobe and the lateral segment of the left lobe. Most authors agree on the predominance of these contrast enhancements at the right lobe-left lobe junction, in segment IV and around the left portal branch [10, 7, 8].

In the presence of superior vena cava obstruction, the left hepatic lobe may occasionally demonstrate areas of focally increased blood flow in the collateral veins that can be seen on images. Typically, the collateral venous pathway includes the internal mammary vein that connects to the left portal vein via the paraumbilical vein $[11,12]$. The flow of blood through the collateral veins may result in areas of focally increased blood flow to the liver [11, 12]. Specifically, an area of increased activity in segment IV of liver (within the medial segment of the left hepatic lobe in what was formerly known as the quadrate lobe) has been well documented in patients with superior vena cava obstruction; this area of increased activity can be seen on $99 \mathrm{mTc}$-sulfur colloid scans of the liver and spleen and is referred to as the focal hepatic hot spot sign [11]. The equivalent of this sign may also be seen on contrast material-enhanced computed tomographic (CT) scans). Some other causes of hepatic hot spots include Budd-Chiari syndrome, liver abscess, hemangioma, focal nodular hyperplasia, and hepatocellular carcinoma $[12,13]$. With the exception of Budd-Chiari syndrome, which causes the hot spot sign to occur in the caudate lobe, all other entities can cause the hot spot sign

\section{CONCLUSION}

The association of hepatic perfusion disorders to the visualization of thoracic and thoraco abdominal diversion pathways should suggest the diagnosis of thrombosis of the VCS, a fortiori in the presence of a superior vena cava syndrome or an upper mediastinal mass.

\section{REFERENCES}

1. Vilgrain.V Maladies vasculaires du foie. Imagerie de l'abdomen. Lavoisier: Médecine-Sciences Publications. 2010: 166-90.

2. Pastacaldi S, Teixeira R, Montalto P, Rolles K, Burroughs AK. Hepatic artery thrombosis after orthotopic liver transplantation: a review of nonsurgical causes. Liver Transpl. 2001; 7:75-81.

3. Sabba C, Pompili M. The hepatic manifestations of hereditary haemorrhagic telangiectasia. Aliment Pharmacol Therap. 2008; 28:523-33.

4. Stanford W, Jolles H, Ell S, Chiu LC. Superior vena cava obstruction: a venographic classification. AJR Am J Roentgenol. 1987; 148:259-62.

5. Qanadli SD, El Hajjam M, Bruckert F, Judet O, Barre O, Chagnon S, Lacombe P. Helical CT phlebography of the superior vena cava: diagnosis and evaluation of venous obstruction. AJR. American journal of roentgenology. 1999 May;172(5):1327-33.

6. Baba Y, Miyazono N, Inoue H, Kanetsuki I, Niwatsukino H, Tanaka D, Nishi H, Nandate T, Ohkubo K, Nakajo M. Altered flow dynamics of intravascular contrast material to the liver in superior vena cava syndrome: CT findings. Abdominal imaging. 2000 Mar 1;25(2):146-50.

7. Attali P, Brauner M, Dumas JL, Safa P, Goldlust D. Trouble de la perfusion hépatique au cours d'un syndrome cave supérieur: à propos d'un cas exploré en tomodensitométrie. J Radiol. 1998; 79:267-9.

8. Bashist B, Parisi A, Frager DH, Suster B. Abdominal CT findings when the superior vena cava, brachiocephalic vein, or subclavian vein is obstructed. AJR Am J Roentgenol. 1996; 167:1457-63. 
9. Dahan H, Arrivé L, Monnier-Cholley L, Le Hir P, Zins M, Tubiana JM. Cavoportal collateral pathways in vena cava obstruction: imaging features. AJR Am J Roentgenol. 1998; 171:140511.

10. Baba Y, Ohkubo K, Nakai H, Hamada K, Hokotate $\mathrm{H}$, Nakajo M. Focal enhanced areas of the liver on computed tomography in a patient with superior vena cava obstruction. Cardiovasc Intervent Radiol. 1999;22:69-70.

11. Yamada T, Takahashi K, Shuke N, Katada R, Mineta M, Nagasawa K, Saitou Y, Aburano T. Focal hepatic" hot spot" in superior vena cava obstruction: correlation between radiocolloid hepatic SPECT and contrast-enhanced CT. Clinical nuclear medicine. 1999 Jul 1;24(7):533-4.

12. Muramatsu T, Miyamae T, Mashimo M, Suzuki K, Kinoshita S, Dohi Y. Hot spots on liver scans associated with superior or inferior vena caval obstruction. Clin Nucl Med. 1994; 19:622- 629.

13. Welch TJ, Sheedy 2nd PF, Johnson CM, Stephens DH, Charboneau JW, Brown ML, May GR, Adson MA, McGill DB. Focal nodular hyperplasia and hepatic adenoma: comparison of angiography, CT, US, and scintigraphy. Radiology. 1985 Sep;156(3):593-5. 